\section{A case report on Meigs' syndrome and elevated serum CA-125: A rare} case report

\section{Divya Khanduja ${ }^{1 *}$ and NC Kajal ${ }^{2}$}

\author{
${ }^{1}$ Junior Resident, Department of Pulmonary Medicine, Government Medical College, Amritsar, \\ Punjab, India \\ ${ }^{2}$ Professor, Department of Pulmonary Medicine, Government Medical College, Amritsar, Punjab, \\ India
}

\section{Abstract}

Meigs syndrome is an uncommon presentation, where a benign ovarian neoplasia presents along with ascites and pleural effusion. About $1 \%$ of ovarian neoplasia can present as Meigs syndrome. Patients with Meigs' syndrome and elevated serum CA-125 are not frequently reported. We report a case of a 50-year-old women who presented with shortness of breath, cough, weight loss of one and half month duration. Chest radiograph of the patient with clinical examination of patient confirms pleural effusion as cause of progressive shortness of breath. The presence of a pelvic mass and elevated serum CA-125, which raised the possibility of malignancy. After complete resection of tumor, the pathologic reports confirmed a benign ovarian neoplasia. We highlight the importance of suspicion, careful general examination, radiological assessment and histological tests to confirm the diagnosis of Meigs' syndrome.

\section{More Information}

*Address for Correspondence: Divya Khanduja, Junior Resident, Department of Pulmonary Medicine, Government Medical College, Amritsar, Punjab, India, Tel: 9582969172; Email: divyakhandujalhmc@gmail.com

Submitted: March 08, 2021

Approved: March 23, 2021

Published: March 24, 2021

How to cite this article: Khanduja D, Kajal NC. A case report on Meigs' syndrome and elevated serum CA-125: A rare case report. J Pulmonol Respir Res. 2021; 5: 031-033.

DOI: 10.29328/journal.jprr.1001021

Copyright: $\odot 2021$ Khanduja D, et al. This is an open access article distributed under the Creative Commons Attribution License, which permits unrestricted use, distribution, and reproduction in any medium, provided the original work is properly cited.

Keywords: Meigs' syndrome; CA-125; Exudative pleural effusion; Benign ovarian neoplasia

(D) Check for updates

(3) Open Access

\section{Introduction}

Meigs syndrome is defined as the presence of pleural effusion and ascites in association with a benign ovarian tumor- Fibroma, thecoma, granulosa cell tumor or Brenner tumor [1]. Some patients have either ascites or pleural effusion with benign ovarian tumors and are classified as Atypical/ Incomplete Meigs syndrome. Meigs syndrome happens in $1 \%$ of all ovarian tumors. It is most commonly associated with ovarian fibroma. Ovarian fibromas are diagnosed in $2 \%$ to $5 \%$ of excised ovarian tumors. Although cases have been reported in women before their third decade, Meigs syndrome is extremely rare in women younger than 30 years old. The syndrome is much more common in postmenopausal women, especially around 50 years, and its peak incidence is in the women in their seven-decade [2,3]. The clinical presentation of a pelvic mass, ascites and pleural effusion is often associated with ovarian carcinoma. Furthermore, if blood and/or pleural fluid CA-125 are elevated, it raises suspicion for malignancy [4]. Herein we report a case which presented with these features and turned out to be a case of benign ovarian tumor.

\section{Case presentation}

A 50-year-old postmenopausal women who presented to the emergency room with complaints of progressively worsening dyspnoea on exertion, dry cough, discomfort over right hemithorax elicited by deep inspiration. She denied weight loss or fever, but admitted to increased abdominal girth in previous 7 to 8 months. There is no history of tuberculosis in past or family. On physical examination, she was slightly tachypnoeic without the use of accessory muscles, with $\mathrm{SpO} 2$ of $90 \%$ on room air. Otherwise, the vital signs were normal. On auscultation, there were absent breath sounds and dullness to percussion over the lower half of the right hemithorax. On abdominal examination, a globular shaped mass of firm consistency, smooth and slightly tender on palpation from the right hypochondrium to the pelvic area. No signs of ascites were evident.

The Chest X-ray showed right sided pleural effusion and bilateral heterogenous opacities. Thoracocentesis was done and the cytological analysis showed the presence of $95 \%$ lymphocytes and $5 \%$ polymorphonuclear structures, negative for malignant cells. Biochemical analysis of fluid examination showed the exudative picture with the proteins- $5.1 \mathrm{~g} / \mathrm{l}$, glucose-21, with ADA-10.2. The Xpert MTB/RIF detects no DNA sequences specific for Mycobacterium tuberculosis in fluid aspiration. The serum level of CA-125 was $534 \mathrm{U} / \mathrm{ml}$. Blood levels of glucose, total protein, creatinine, aspartate 
aminotransferase, and alanine aminotransferase were normal. Mantoux test, serology of HIV were negative. AFB culture of sputum was negative.

CT scan of abdomen showed a $17.7 \times 13$ x $7.9 \mathrm{~cm}$ abdominopelvic heterogenous mass with clear borders without lymphadenopathy was observed. The patient underwent a hysterectomy and bilateral oophorectomy. A solid- cystic smooth surfaced and highly vascular mass dependant of both right and left adnexa. Scanty amount of peritoneal fluid was present. The intraoperative pathology of mass was suggestive of ovarian fibroma. Few months later, the patient reported no symptoms, had normal lung sounds, and a normal chest X-ray (Figures 1,2).

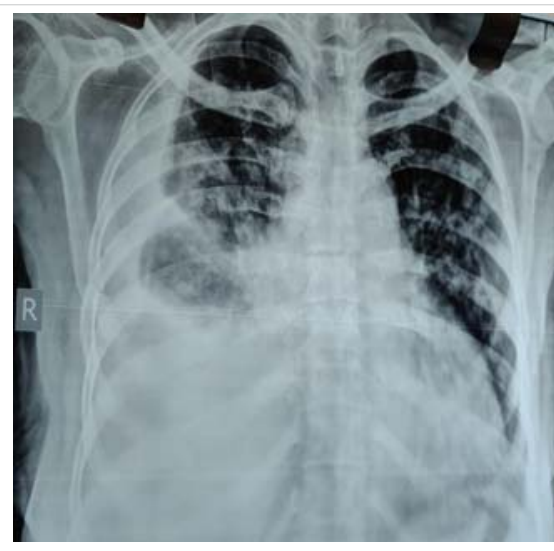

Figure 1: Chest $\mathrm{X}$-ray showing right side pleural effusion and bilateral heterogenous opacities.

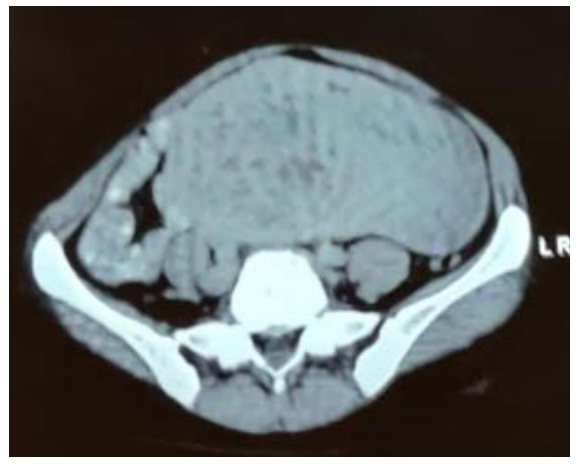

Figure 2: CT scan of abdomen showing a $17.7 \times 13 \mathrm{~cm}$ abdominopelvic heterogenous mass with clear borders, appears to originate from right adnexa, without lymphadenopathy.

\section{Discussion}

Meigs' syndrome was first reported in 1934, where removal of the fibroma resulted in the elimination of ascites and pleural effusion. Based on the historical determinants, case reports, and review articles, four criteria should be met to classify a case as classic Meigs' syndrome. These include: (a) benign fibroma (they account for up to $2 \%$ of resected ovarian neoplasms [5], or the less common fibroma-like (thecoma, granulosa cell tumor, or Brenner tumor) ovarian tumors, (b) ascites, (c) pleural effusion, and (d) resolution of ascites and pleural effusion after removal of the tumor [4].
However, some authors also accept as Meigs' syndrome any case of a low-grade malignant tumor associated with ascites and pleural effusion that disappear after surgical removal of the tumor [6]. The terminology may be somehow confusing, as Demons' syndrome refers to all benign genital tumors, Demons-Meigs' eponym is reserved for the description of ovarian fibromas and granulosa cell tumors, and Demons' pseudo-syndrome includes all other entities [7]. Moreover, Pseudo-Meigs' syndrome, a clinical syndrome of pleural effusion, ascites, and an ovarian mass that is not a fibroma or fibroma-like mass/tumor, has been reported from a number of sources, particularly leiomyomas, struma ovarii, mucinous cystadenoma, teratoma, and malignancies that are metastatic to the ovary (particularly colorectal cancer) [8].

Meigs' syndrome presents more often in the fifth decade of life.Most of the patients ofMeigs' syndromepresentwith fatigue, shortness of breath, increased abdominal girt, weight gain or weight loss, non-productive cough, bloating, amenorrhoea in pre-menopausal women, menstrual irregularity. The cause of ascitic fluid appears to be a generalized secretion by the tumor itself. Moreover, the stimulation of the peritoneum's mesothelial cells by the release of vascular endothelial growth factor (VEGF), IL-1, IL-6, IL-8, and TNF- $\alpha$ from the tumor is reported as another cause of the exudation. Only large tumors are frequently associated with ascites, which may not be detectable on physical examination, and the amount of ascites does not influence the amount of pleural effusion that develops. The genesis of the pleural effusion is same as in patients with hepatic hydrothorax, through pores in the diaphragm, and the similar characteristics between peritoneal and pleural fluid and the rapid re-accumulation of pleural fluid after evacuation supports this hypothesis. However, some authors believe that the pleural fluid arises from the transdiaphragmatic transfer of ascitic fluid by the lymphatic vessels, which seem to be larger on the right side, potentially explaining the finding of a right pleural effusion seen in approximately $70 \%$ of patients, being left-sided in $10 \%$, and bilateral in $20 \%$. Based on the protein levels, the effusion is usually an exudate with a low white blood cell count (fewer than $1000 / \mathrm{mm}^{3}$ ) and is occasionally bloody [6]. In the present case, the pleura effusion was on the right side and exudative in nature as seen in majority of cases.

- Pleural fluid is classified as a transudate or exudate based on modified Light's criteria. Pleural fluid is considered an exudative effusion if at least one of the criteria are met $[9,10]$. Pleural fluid protein/serum protein ratio more than 0.5

- Pleural fluid lactate dehydrogenase (LDH)/serum LDH ratio of more than 0.6

- Pleural fluid LDH is more than two-thirds of the upper limits of normal laboratory value for serum LDH

Pleural effusion is common clinical entity in day-to-day practice in India. Tuberculosis is one of the common causes of 
exudative effusions along with parapneumonic effusions and malignancy. Pleural fluid ADA levels is highly sensitive with good specificity for the diagnosis of etiology of tubercular effusions [11]. Meigs' syndrome is a rare disease characterized by a triad of pleural effusion, ascites and ovarian tumors. Meigs' syndrome is a rare case of exudative pleural effusion may be misdiagnosed as Tuberculosis in country like India where Tuberculosis is an endemic disease. However, a clinical diagnosis of Meigs' syndrome remains challenging because pleural effusion and ascites can be common findings in a variety of underlying conditions. Furthermore, these findings can be misdiagnosed as pleural and peritoneal dissemination caused by potentially malignant tumors, leading to the administration of improper treatment [12].

A CA-125 test is not accurate enough to use for the ovarian cancer screening in general because many noncancerous conditions can increase the serum level, including normal conditions such as menstruation and pregnancy, and noncancerous conditions such as cirrhosis of the liver, uterine fibroid or endometriosis. Besides ovarian cancer, certain cancers may also cause an increased level of CA-125, including endometrial, peritoneal and fallopian tube cancers [13] while active smoking seems to decrease it [14] Women with Meigs' syndrome may also have markedly elevated serum CA-125. Therefore, this finding should not be taken as an unequivocal sign of malignancy [4,6,15-17].

\section{Conclusion}

The diagnosis of Meigs' syndrome should be entertained in all women with a pelvic mass, ascites and pleural effusion that present with a negative fluid cytology, and it is confirmed by the finding of a benign ovarian tumor and the nonrecurrence of peritoneal and pleural effusion once the mass is removed. It is important to be aware that, a high serum and/or fluid CA125 does not compulsorily indicate malignancy.

\section{References}

1. Riker D, Goba D. Ovarian mass, pleural effusion, and ascites: revisiting Meigs syndrome. J Bronchology Interv Pulmonol. 2013; 20: 48-51. PubMed: https://pubmed.ncbi.nlm.nih.gov/23328144/

2. Saha S, Robertson M, Meigs' and Pseudo-Meigs' syndrome. Australas J Ultrasound Med. 2012; 15: 29-31.

PubMed: https://www.ncbi.nlm.nih.gov/pmc/articles/PMC5025132/

3. Shiau CS, Chang MY, Hsieh CC, Hsieh TT, Chiang CH. Meigs' syndrome in a young woman with a normal serum CA-125 level. Chang
Gung Med J. 2005; 28: 587-591

PubMed: https://pubmed.ncbi.nlm.nih.gov/16265850/

4. Kwan $\mathrm{BCH}$, Light RW. Effusions associated with gynecologic and obstetric conditions. In: Textbook of Pleural Diseases, 3rd Ed.. Light RW, Gary Lee YC (ed): CRC Press, Taylor \& Francis Group. 2016; 427: 39.

5. Quirk JT, Natarajan N. Ovarian cancer incidence in the United States, 1992-1999. Gynecol Oncol. 2005; 97: 519-523.

PubMed: https://pubmed.ncbi.nlm.nih.gov/15863154/

6. Krenke R, Maskey-Warzechowska M, Korczynski P, ZielinskaKrawczyk M, Klimiuk J, et al. Pleural effusion in Meigs' syndrome transudate or exudate?: systematic review of the literature. Medicine (Baltimore). 2015; 94: 2114.

PubMed: https://www.ncbi.nlm.nih.gov/pmc/articles/PMC5008483/

7. Brun JL. Demons syndrome revisited: a review of the literature. Gynecol Oncol. 2007; 105: 796-800.

PubMed: https://pubmed.ncbi.nlm.nih.gov/17433421/

8. Peparini N, Chirletti P. Ovarian malignancies with cytologically negative pleural and peritoneal effusions: demons' or Meigs' pseudosyndromes? Int J Surg Pathol. 2009; 17: 396-397. PubMed: https://pubmed.ncbi.nlm.nih.gov/19448068/

9. Bedawi EO, Hassan M, Rahman NM. Recent developments in the management of pleural infection: A comprehensive review. Clin Respir J. 2018; 2: 2309-2320.

PubMed: https://pubmed.ncbi.nlm.nih.gov/30005142/

10. Lepus CM, Vivero M. Updates in Effusion Cytology. Surg Pathol clin. 2018; 11: 523-544.

PubMed: https://pubmed.ncbi.nlm.nih.gov/30190139/

11. Reddy SL, Varaprasad K, Narahari N, Bhaskar K, Varma GR, et al. Clinical and etiological profile of an exudative pleural effusion in a tertiary care center. Indian J Respir Care. 2019; 8: 22-26. http://www.ijrc.in/text.asp?2019/8/1/22/249355

12. Murayma. BMC Cardiovascular Disorders.

13. CA 125 test. 2020. https://www.mayoclinic.org/tests-procedures/ca125-test/about/pac-20393295

14. Johnson CC, Kessel B, Riley TL, Ragard LR, Williams CR, et al. The epidemiology of CA-125 in women without evidence of ovarian cancer in the prostate, lung, colorectal and ovarian cancer (PLCO) screening trial. Gynecol Oncol. 2008; 110: 383-389.

PubMed: https://pubmed.ncbi.nlm.nih.gov/18586313/

15. Kortekaas KE, Pelikan H. Hydrothorax, ascites and an abdominal mass: not always signs of a malignancy - three cases of Meigs' syndrome. Radiol Case. 2018; 12: 17-26.

PubMed: https://pubmed.ncbi.nlm.nih.gov/29875983/

16. Taniguchi $\mathrm{Y}$, Nishikawa H, Maeda N, Terada $\mathrm{Y}$. Breathlessness, pleural effusions, fibromas, and Meigs syndrome: look beyond the chest and don't delay! Lancet. 2020; 395: 32.

PubMed:https://pubmed.ncbi.nlm.nih.gov/32061301/

17. Sofoudis C, Kouiroukidou P, Louis K, Karasaridou K, Toutounas K, et al Enormous ovarian fibroma with elevated $\mathrm{Ca}-125$ associated with Meigs' syndrome. Presentation of a rare case. Eur J Gynaecol Oncol. 2016; 37: 142-143.

PubMed: https://pubmed.ncbi.nlm.nih.gov/27048129/ 